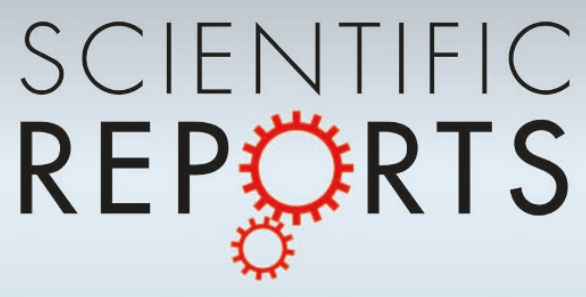

OPEN

SUBJECT AREAS:

TWO-DIMENSIONAL

MATERIALS

CHEMICAL PHYSICS

ELECTRONIC PROPERTIES AND MATERIALS

Received

25 March 2014

Accepted

14 May 2014

Published

30 May 2014

Correspondence and requests for materials should be addressed to J.T.H. (jthan@keri.re. kr) or G.-W.L. (gwleephd@keri.re.kr)

\section{Extremely Efficient Liquid Exfoliation and Dispersion of Layered Materials by Unusual Acoustic Cavitation}

Joong Tark Han ${ }^{1,2}$, Jeong In Jang ${ }^{1}$, Haena Kim ${ }^{4}$, Jun Yeon Hwang ${ }^{5}$, Hyung Keun Yoo ${ }^{6}$, Jong Seok Woo ${ }^{3}$, Sua Choi ${ }^{3}$, Ho Young Kim ${ }^{3}$, Hee Jin Jeong ${ }^{3}$, Seung Yol Jeong ${ }^{3}$, Kang-Jun Baeg ${ }^{3}$, Kilwon Cho ${ }^{4}$ \& Geon-Woong Lee ${ }^{3}$

\begin{abstract}
'Multidimensional Nanomaterials Research Group, Korea Electrotechnology Research Institute (KERI), Changwon 642-120, Republic of Korea, ${ }^{2}$ Department of Electrical Functionality Material Engineering, Korea University of Science and Technology (UST), Daejon, 305-333, Republic of Korea, ${ }^{3}$ Nano Carbon Materials Research Group, Korea Electrotechnology Research Institute (KERI), Changwon 642-120, Republic of Korea, ${ }^{4}$ Department of Chemical Engineering, Pohang University of Science and Technology (POSTECH), Pohang, 790-784, Republic of Korea, ${ }^{5}$ Institute of Advanced Composite Materials, Korea Institute of Science and Technology (KIST), Eunha-ri san 101, Bondong-eup, Wanju-gun, Jeolabuk-do, 565-905, Republic of Korea, ${ }^{6}$ Nanophotonics Laboratory, Advanced Photonics Research Institute, 123 Cheomdan-gwagiro, Buk-gu, Gwangiu 500-712, Republic of Korea.
\end{abstract}

Layered materials must be exfoliated and dispersed in solvents for diverse applications. Usually, highly energetic probe sonication may be considered to be an unfavourable method for the less defective exfoliation and dispersion of layered materials. Here we show that judicious use of ultrasonic cavitation can produce exfoliated transition metal dichalcogenide nanosheets extraordinarily dispersed in non-toxic solvent by minimising the sonolysis of solvent molecules. Our method can also lead to produce less defective, large graphene oxide nanosheets from graphite oxide in a short time (within $10 \mathrm{~min}$ ), which show high electrical conductivity $\left(>20,000 \mathrm{~S} \mathrm{~m}^{-1}\right)$ of the printed film. This was achieved by adjusting the ultrasonic probe depth to the liquid surface to generate less energetic cavitation (delivered power $\sim 6 \mathrm{~W}$ ), while maintaining sufficient acoustic shearing $\left(0.73 \mathrm{~m} \mathrm{~s}^{-1}\right)$ and generating additional microbubbling by aeration at the liquid surface.

U ltrasonic acoustic cavitation has been used in many applications such as exfoliation and dispersion of nanomaterials, sonochemical synthesis, ultrasonic cleaning, laser surgery in medicine, and lithotripsy because acoustic cavitation owes its usefulness to the concentration of acoustic energy in small volumes ${ }^{1-7}$. In particular, acoustic cavitation has been utilised in the production of two-dimensional nanosheets such as graphene, transition metal dichalcogenides, and transition metal oxides from bulk layered crystal materials in liquid or debundling of nanotubes in solution ${ }^{8-12}$. Usually, this process uses the physical effects of high energetic transient acoustic cavitation: the formation, growth, and implosive collapse of bubbles at high ultrasonic intensities $\left(10-30 \mathrm{~W} \mathrm{~cm}^{-2}\right)$ in a liquid medium ${ }^{1}$. However, the high-speed jets and intense shock waves induced by transient cavitation can diminish the size of the nanosheets or generate defects on the surface, which decreases their electrical and other useful properties. Furthermore, highly reactive radicals and by-products formed by the sonolysis of organic solvent molecules can unexpectedly influence the dispersion of nanomaterials in organic solvents considering the surface tension and the solubility parameters of the solvent. In this context, the less energetic 'stable' acoustic cavitation formed at fairly low ultrasonic intensities $\left(1-3 \mathrm{~W} \mathrm{~cm}^{-2}\right)$ is advantageous in giving a low energy to nanomaterials and minimizing the sonolysis of organic materials. Usually, stable acoustic cavitation can be generated by reducing the intensity adjusting amplitude, while the low amplitude also decreases acoustic shearing of the solution ${ }^{13}$. However, for the effective exfoliation and dispersion of layered materials, highrate shearing in the solution is very helpful ${ }^{14}$. Homogenisers are well known tools for generating the shear force in solution; however, they cannot generate an energetic cavitation bubble and require considerable time (several hours) to fully exfoliate layered materials such as graphite oxide ${ }^{14}$. If the less energetic acoustic cavitation and high shearing can be applied to the target solution at the same time, the layered materials would be well exfoliated and dispersed in a short time. This process would minimise the defect formation and the cracking of two-dimensional (2D) nanosheets or nanotubes without sonolysis of the solvent molecules or dispersant. 
a

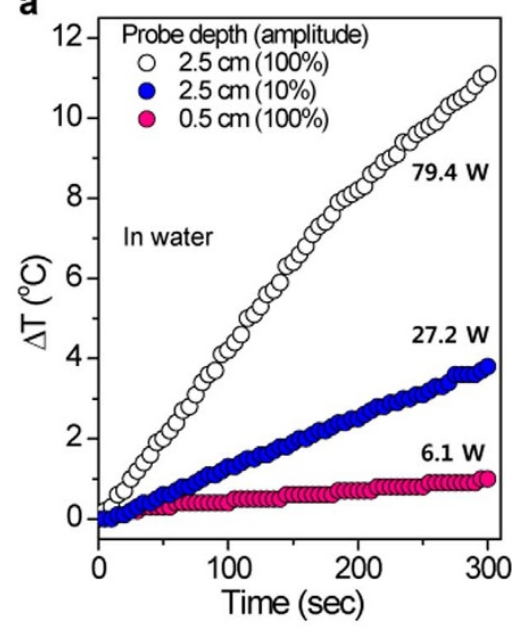

C

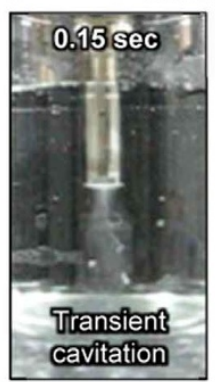

Depth $=2.5 \mathrm{~cm}$

In water (amplitude 100\%)

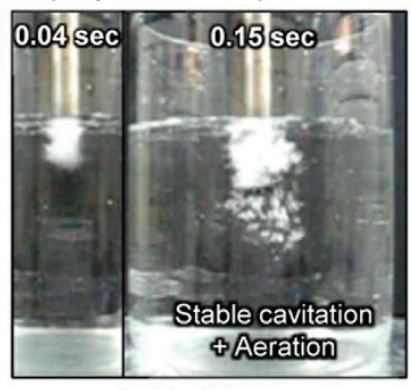

Depth $=0.5 \mathrm{~cm}$ b

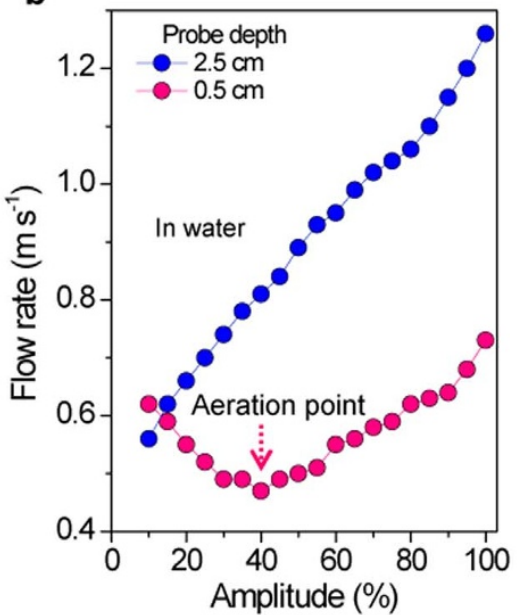

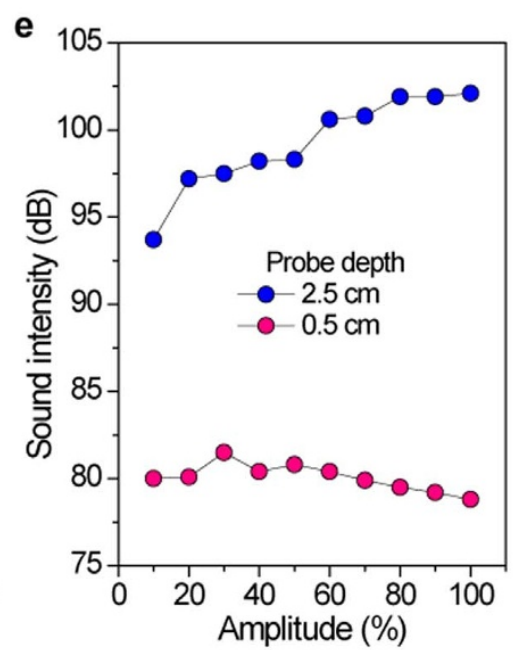

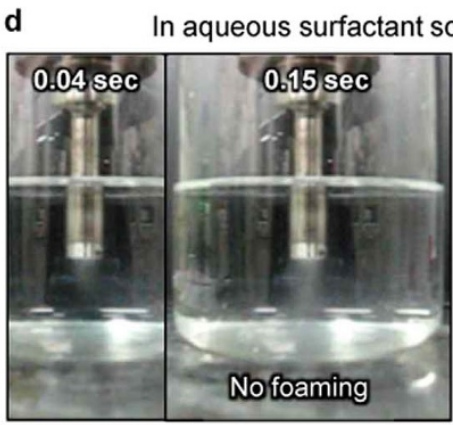

Depth $=2.5 \mathrm{~cm}$

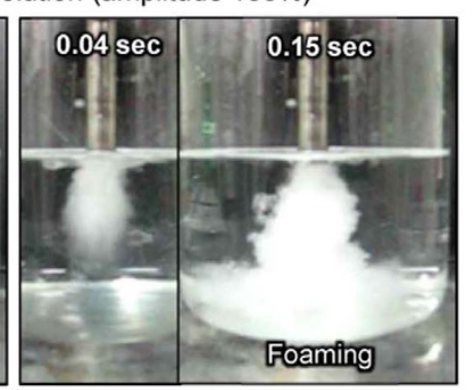

Depth $=0.5 \mathrm{~cm}$

Figure 1 Characterisation of ultrasonic acoustic cavitation. (a) Temperature change $(\Delta T)$ over time and (b) Flow rate as a function of amplitude setting during the probe sonication of pure water by varying the probe depth and amplitude. (c and d) Photographs of the acoustic cavitation bubbles in water (c) and in aqueous sodium dodecylbenzenesulphate solution (d) during probe sonication at different probe positions at $100 \%$ amplitude after 0.15 sec. (Titanium probe tip, $12.7 \mathrm{~mm}$ ). (e) Acoustic sound intensity as a function of the amplitude setting at different probe depths in the aqueous surfactant solution.

Here we demonstrate the extraordinary dispersion and exfoliation of $2 \mathrm{D}$ nanosheets in solution. The combination of ultrasonic acoustic cavitation maintaining sufficient acoustic shearing and additional microbubbling by aeration allows us to produce extraordinarily dispersed transition metal dichalcogenide nanosheets in non-toxic solvents while minimising the sonolysis of the solvent molecules. In addition, in an extremely short time $(10 \mathrm{~min})$, we produced large $(>10 \mu \mathrm{m})$ chemically modified graphene nanosheets from graphite oxide with fewer defects than those produced by other methods without requiring further separation processes. This extremely efficient method was simply achieved by moving the ultrasonic probe to the surface of the liquid.

\section{Results}

Generation of the less energetic acoustic cavitation, shearing and foaming. Using conventional flat tip probes with a $12.7-\mathrm{mm}$ diameter, we first investigated the influence of ultrasound parameters (amplitude, time, and probe immersion depth) on the delivered power (related to temperature change $(\Delta T))^{15}$, the acoustic flow rate, and the bubbling behavior in $200 \mathrm{~mL}$ water (Supplementary Fig. 1). At $100 \%$ amplitude, a high power of $80 \mathrm{~W}$ was delivered at the probe depth of $2.5 \mathrm{~cm}$. At $10 \%$ amplitude, the power was decreased to $30 \mathrm{~W}$. This minimum power is higher than that for the less energetic stable cavitation. However, by adjusting the probe depth to $0.5 \mathrm{~cm}$ from the liquid surface, we could minimise the delivered power to $6 \mathrm{~W}$ even at $100 \%$ amplitude due to the considerable increase in acoustic resistance at the liquid surface (Fig. 1a). This delivered power is enough to minimise the highly energetic transient cavitation, forming less energetic cavitation ${ }^{13}$. In addition, the acoustic flow rates at the probe depth of $2.5 \mathrm{~cm}$ were linearly increased from 0.56 to $1.26 \mathrm{~m} \mathrm{~s}^{-1}$ by adjusting the amplitude from $10 \%$ to $100 \%$. Importantly, at the probe depth of $0.5 \mathrm{~cm}$, the acoustic flow rate decreased from 0.62 to $0.47 \mathrm{~m} \mathrm{~s}^{-1}$ and then increased to $0.73 \mathrm{~m} \mathrm{~s}^{-1}$ at $100 \%$ amplitude, which is faster than the $10 \%$ amplitude at $2.5 \mathrm{~cm}$ probe depth (Fig. 1b). Bubbling at the $0.5 \mathrm{~cm}$ probe depth is another interesting phenomenon due to aeration by the liquid surface instability under acoustic oscillation (Fig. 1c) ${ }^{16}$. Interestingly, the bubbling started at $40 \%$ amplitude. Bubbling by aeration at the liquid surface can be helpful in the dispersion of nanomaterials because the bubbling can produce a greater shearing effect on the particles in suspension under a less energetic acoustic flow $^{14}$. Notably, this bubbling was more clearly observed in a surfactant (sodium dodecylbenzenesulphate) solution, inducing long-lived foaming (Fig. 1d and Supplementary Fig. 2). In terms of safety, the microbubble formation can reduce hazardous acoustic sound (Fig. 1e). However, for a probe depth of $2.5 \mathrm{~cm}$, we could not observe long-lived foaming even in surfactant solutions because of the bubble collapse.

Liquid exfoliation and dispersion of $\mathrm{MoS}_{2}$. This less energetic and unusual acoustic cavitation phenomenon was applied in the exfoliation of $\mathrm{MoS}_{2}$, a typical layered transition metal dichalcogenide material. The exfoliation and dispersion of transition metal dichalcogenide nanosheets in non-toxic common solvents in high concentration is important for applications in nanocomposites, electrochemical electrodes or electronics. Basically, the liquid exfoliation of bulk 

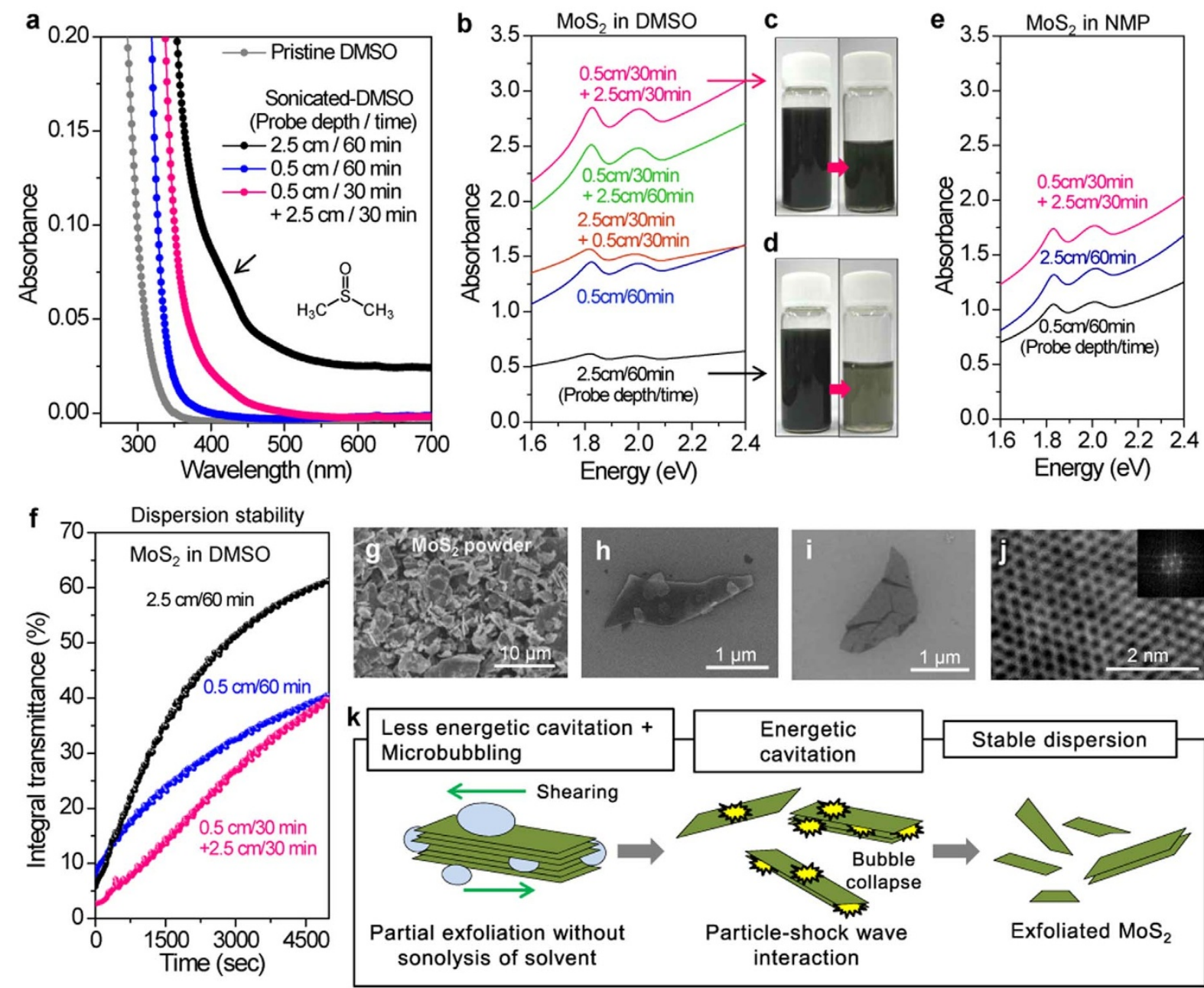

Figure $2 \mid$ Liquid exfoliation and dispersion of $\mathrm{MoS}_{2}$. (a) UV-vis absorbance of DMSO and NMP before and after sonication varying the probe immersion depth, respectively. (b) Absorption spectra of $\mathrm{MoS}_{2}$ nanosheet dispersions in DMSO. (c-d) Photographs of the dispersion of MoS 2 in DMSO prepared by sequential step-by-step sonication at (c) $0.5 \mathrm{~cm}$ and $2.5 \mathrm{~cm}$ probe depths for $30 \mathrm{~min}$ each, and at (d) $2.5 \mathrm{~cm}$ probe depth for 60 min before (left) and after (right) centrifugation at $1500 \mathrm{rpm}$. (e) Absorption spectra of $\mathrm{MoS}_{2}$ nanosheet dispersions in NMP. (f) Sedimentation measurements for $\mathrm{MoS}_{2}$ in DMSO prepared by sonication at $2.5 \mathrm{~cm}$ probe depth for $60 \mathrm{~min}$, by sonication at $0.5 \mathrm{~cm}$ probe depth for $60 \mathrm{~min}$, and by sequential step-by-step sonication at $0.5 \mathrm{~cm}$ and $2.5 \mathrm{~cm}$ probe depths for $30 \mathrm{~min}$ each. (g-i) FESEM images of (g) a powder, (h) a multilayer, and (i) a very thin and wrinkled nanosheet of $\mathrm{MoS}_{2}$. (j) High resolution TEM image of $\mathrm{MoS}_{2}$ monolayer and (inset) fast Fourier transform of the image. (k) Schematic illustration of highly concentrated dispersion of layered compounds by sequential and repeating adjustment of the sonication probe at $0.5 \mathrm{~cm}$ from the liquid surface (less energetic cavitation, acoustic shearing and microbubbling by aeration) and at $2.5 \mathrm{~cm}$ (energetic transient cavitation).

$\mathrm{MoS}_{2}$ crystals in common solvents can be done by considering the surface tension and the Hansen solubility parameters. It has been reported that dimethylsulphoxide (DMSO) is a less dispersible solvent for $\mathrm{MoS}_{2}$ exfoliation than pyrrolidone derivative solvents when conventional probe sonication is applied ${ }^{10}$. However, the DMSO molecules can be easily decomposed by energetic transient acoustic cavitation, generating various radicals such as $\mathrm{CH}_{3} \mathrm{SO} \bullet$, $\mathrm{CH}_{3} \mathrm{SO}_{2} \bullet, \mathrm{CH}_{3} \mathrm{SOCH}_{2} \bullet$ and $\mathrm{CH}_{3} \bullet$ during the conventional probe sonication, while the DMSO molecules can act like free radical ${ }^{17}$. Therefore, we applied a less energetic cavitation method for the exfoliation dispersion of $\mathrm{MoS}_{2}$ in DMSO. Before the exfoliation process, the DMSO was sonicated by itself by adjusting the probe depth and time. Ultraviolet-visible (UV-vis) spectra of the fresh and sonicated DMSO clearly show that the DMSO molecules were severely decomposed by sonication at a $2.5 \mathrm{~cm}$ probe depth, showing peak near $450 \mathrm{~nm}$ (Fig. 2a). Furthermore, the surface tension data clearly showed a decrease in the surface tension (Supplementary Fig. 3). Notably, these results indicate that the crucial solubility parameter affecting the dispersion of $\mathrm{MoS}_{2}$ can be changed by acoustic cavitation. However, importantly, probe sonication at the liquid surface minimised the sonolysis of the DMSO. The spectroscopic data obtained thus far allowed us to hypothesise that the reported concentration of the exfoliated $\mathrm{MoS}_{2}$ in DMSO can be increased because the surface tension of $\mathrm{MoS}_{2}$ is larger than DMSO. In fact, the exfoliated $\mathrm{MoS}_{2}$ nanosheets and flakes were less dispersible in DMSO than in $N$-methyl pyrrolidone (NMP) by sonication at a $2.5 \mathrm{~cm}$ probe depth for $60 \mathrm{~min}$ followed by centrifugation at $1500 \mathrm{rpm}$ (Fig. 2b), as previously reported ${ }^{10}$. Notably, by adjusting the ultrasonic probe depth to the liquid surface, the optical absorption of the $\mathrm{MoS}_{2}$ suspension in DMSO dramatically increased and became as high as that in NMP in spite of the less energetic cavitation condition (Fig. $2 b$ to d). However, in NMP, we could not observe this enhancement of dispersibility of the $\mathrm{MoS}_{2}$ after sonication at a $0.5 \mathrm{~cm}$ probe depth (Fig. 2e). The solubility parameter matching in NMP was dominant for dispersion because of the higher thermal stability of NMP over DMSO (Fig. 2a) ${ }^{18}$. Thus, we could obtain high-concentration suspensions of $\mathrm{MoS}_{2}$ in both DMSO and NMP using step-by-step sonication at $0.5 \mathrm{~cm}$ and $2.5 \mathrm{~cm}$ probe depth for $30 \mathrm{~min}$, sequentially. The concentration of $\mathrm{MoS}_{2}$ was thus increased by two times (Fig. 2b). This concentration 


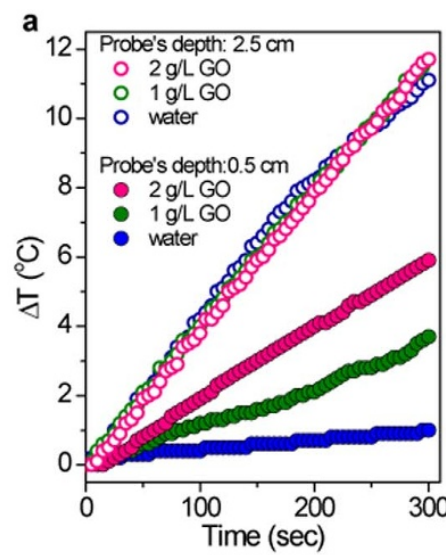

b

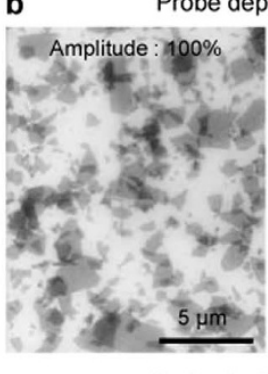

c
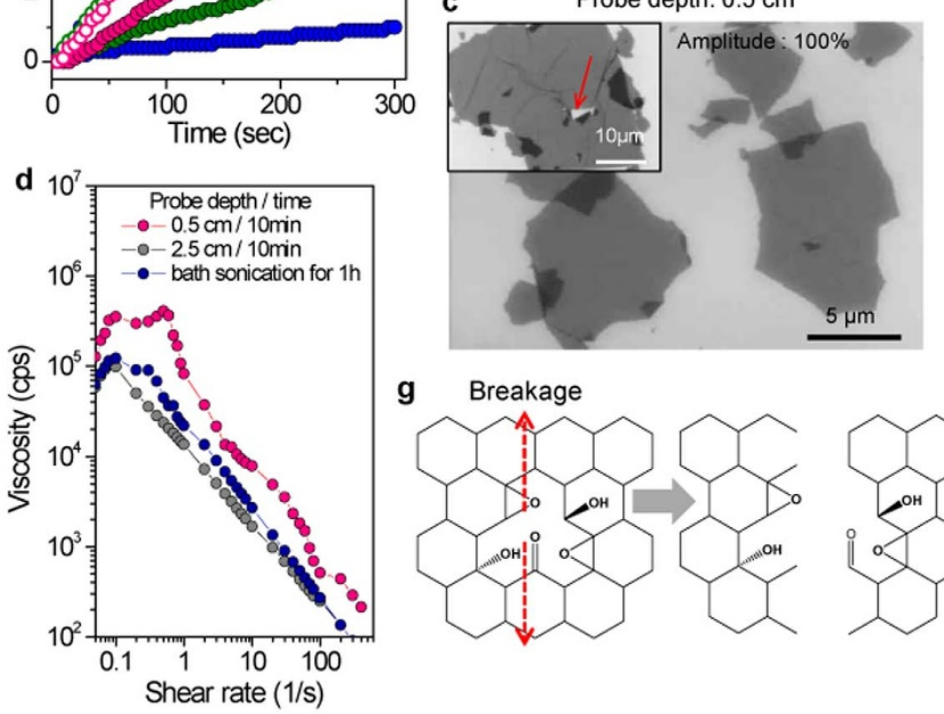

g

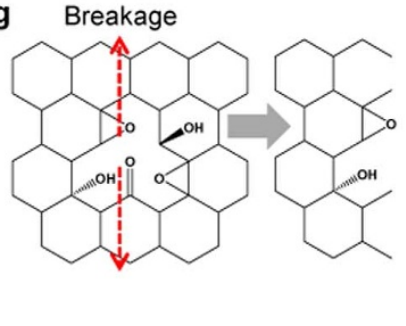

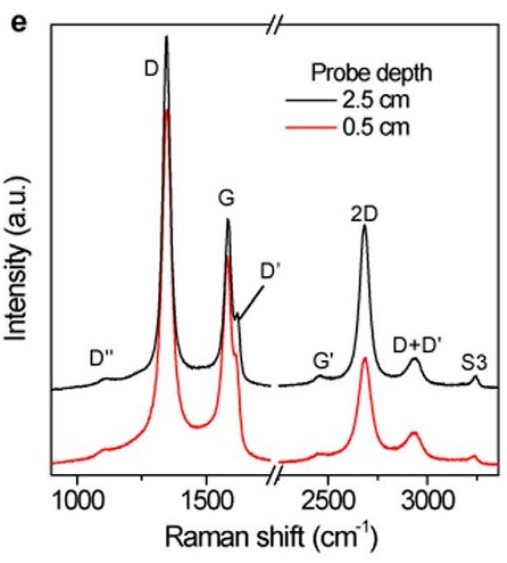

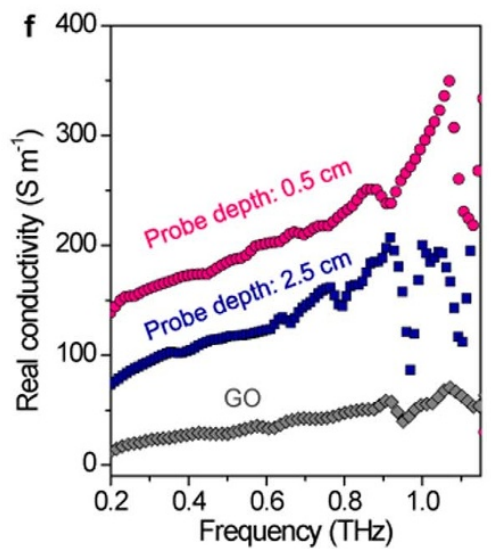

Figure 3 Liquid exfoliation of graphite oxide. (a) Temperature change ( $\Delta T$ ) over time during the probe sonication of pure water and a GO suspension containing different initial amounts of graphite oxide. (b, c) FESEM images of GO nanosheets fabricated by probe sonication at (b) $2.5 \mathrm{~cm}$ and (c) $0.5 \mathrm{~cm}$ probe depths for $10 \mathrm{~min}$. The inset FESEM image in (c) shows the maximum size of GO nanosheet, in which red arrow indicates the initiation of the breakage of GO nanosheet. (d) Shear viscosity of GO pastes prepared at different sonication conditions. (e) Raman spectra of the chemically reduced GO nanosheets fabricated by probe sonication with probe depths of 2.5 and $0.5 \mathrm{~cm}$. (f) Schematic illustration of the GO nanosheet breakage from the defect site or from the $\mathrm{sp}^{3}$ hybridised region during probe sonication. (g) Real THz conductivity of GO, SRGO, and LRGO film.

of exfoliated $\mathrm{MoS}_{2}$ in DMSO is 4.5 times larger than that produced in DMSO by conventional probe sonication and two times larger than that produced in NMP. This result is consistent with a small increase of UV-vis absorption of DMSO without $\mathrm{MoS}_{2}$. However, further sonication at $2.5 \mathrm{~cm}$ decreased the optical absorbance of the $\mathrm{MoS}_{2}$ suspension in DMSO, which clearly demonstrates the detrimental effect of the decomposed DMSO products on the dispersion of the $\mathrm{MoS}_{2}$ nanosheets. Moreover, sequential sonication at $2.5 \mathrm{~cm}$ and $0.5 \mathrm{~cm}$ for $30 \mathrm{~min}$ did not produce a synergistic effect on the dispersion of $\mathrm{MoS}_{2}$ in DMSO. By measuring the integral transmission under centrifugation at $1500 \mathrm{rpm}$, the dispersion stability test demonstrates that the less energetic cavitation is more helpful for long dispersion of the exfoliated $\mathrm{MoS}_{2}$ in DMSO than energetic transient cavitation (Fig. $2 \mathrm{f}$ and Supplementary Fig. 4). In addition, after centrifugation at different speeds, we could observe the distinct advantages of our method in the dispersion of exfoliated $\mathrm{MoS}_{2}$ nanosheets in organic solvents (Supplementary Fig. 5). Transmission electron microscopy (TEM) and scanning electron microscopy (SEM) images show 2D flakes consisting of thin nanosheets (Fig. $2 \mathrm{~h}$ to $\mathrm{j}$ ). Therefore, we believe that less energetic cavitation combined with aeration bubbling contributes to the minimisation of the sonolysis of solvent molecules, leading to a highly concentrated dispersion of exfoliated $\mathrm{MoS}_{2}$ nanosheets and thin flakes. Following the less energetic cavitation and bubbling method with transient cavitation promotes further exfoliation of the thin $\mathrm{MoS}_{2}$ flakes by particle-shock wave interactions as illustrated in Figure $2 \mathrm{k}$, because solvent molecules that are not decomposed can interact with $\mathrm{MoS}_{2}$ surfaces. The peak intensity decrease in the X-ray diffraction patterns of exfoliated $\mathrm{MoS}_{2}$ nanosheets also show the effectiveness of the stepwise exfoliation of $\mathrm{MoS}_{2}$ flakes (Supplementary Fig. 6). In addition, our unusual sonication was also applied in the exfoliation of $\mathrm{WS}_{2}$ in DMSO, in which we also obtained highly dispersed $\mathrm{WS}_{2}$ nanosheets in DMSO by moving the probe to the liquid surface (Supplementary Fig. 7 and 8).

Liquid exfoliation of nanocarbon materials. For further demonstration, we performed the exfoliation of graphite oxide into graphene oxide (GO) nanosheets in aqueous solution. Interestingly, at the energetic cavitation condition (probe depth: $2.5 \mathrm{~cm}$ ), the $\Delta T$ in the GO suspension was not significantly different from that of pure water because the highly energetic transient acoustic cavitation is the main energy source (Fig. 3a). The slight reduction of GO nanosheets at a $2.5 \mathrm{~cm}$ probe depth also shows the temperature increase by the energetic cavitation in water (Supplementary Fig. 9). However, at the less energetic acoustic cavitation condition (probe depth: $0.5 \mathrm{~cm})$, the $\Delta T$ was dominant at the high concentration of graphite oxide in the liquid. This may depend on the pre-trapped gas in the graphite oxide where the bubble nucleation takes place and on the aeration-enhanced bubbling in the presence of solid particles. This high concentration of bubbles can induce fluidic friction between the graphite oxide particles, resulting in shearing, which may increase the temperature, as we can also see in the mechanical homogeniser system. Figure $3 b$ and $c$ shows the striking effect of probe sonication by adjusting the probe depth to achieve fabrication of less defective GO nanosheets. At a probe depth of $2.5 \mathrm{~cm}$, the maximum size of GO was less than $5 \mu \mathrm{m}$ even at $10 \%$ amplitude 
after $10 \mathrm{~min}$ sonication (Fig. 3b). This is due to breakage in the stretched $\mathrm{C}-\mathrm{C}$ bonds or $\mathrm{C}-\mathrm{O}-\mathrm{C}$ bonds ${ }^{19,20}$ by the high energetic physical phenomena of microjets and shock waves ${ }^{3}$. However, at a probe depth of $0.5 \mathrm{~cm}$, the GO nanosheets reached a maximum size of $30 \mu \mathrm{m}$ even at the high output power setting (amplitude 100\%) without further centrifugal separation because of the reduced physical effects (Fig. 3c). At this probe depth, the breakage of GO nanosheets into small pieces $(<1 \mu \mathrm{m})$ was minimised even at low concentration and after long sonication (Supplementary Figs 10 to 12). The combination of less energetic cavitation, acoustic shearing, and bubbling by aeration may lead to less defective exfoliation of graphite oxide in a short time. For comparison, we applied bath sonication with no shearing. After $10 \mathrm{~min}$, the partially exfoliated graphite oxide by bath sonication was more easily precipitated than GO nanosheets produced by probe sonication, and the sizes of GO nanosheets were smaller than those exfoliated by probe sonication at $0.5 \mathrm{~cm}$ probe depth (Supplementary Fig. 13). We also observed distinct shear thickening in the low shear regime for large GO nanosheet paste because of the strong sheet-to-sheet interaction (Fig. 3d). Most importantly, in the Raman spectra (Fig. 3e), the larger $I_{2 D} / I_{G}(\sim 0.98)$ of the small reduced graphene oxide (SRGO) nanosheets than that $(0.53)$ of the large reduced graphene oxide (LRGO) nanosheets indicates that the breakage of GO nanosheets at the energetic cavitation condition was initiated from defects formed during the oxidation of the graphite as illustrated in Figure 3f. Moreover, a high-intensity and narrow (full width at half maximum $\sim 51 \mathrm{~cm}^{-1}$ ) 2D band Raman peak of the RGO nanosheets indicates that the GO nanosheets used in this study have a more ideal graphitic structure even after oxidation ${ }^{21}$. Notably, the electrical conductivity reaches $\sim 20,000 \mathrm{~S} \mathrm{~m}^{-1}$ for a printed LRGO film with RGO pastes stabilised by quadruple hydrogen bonding (QHB) networks (Supplementary Fig. 14), which is similar to that prepared by a homogeniser for $1 \mathrm{~h}^{22}$. However, the small RGO did not form a stable dispersion in the paste because of the lack of QHB interconnectivity (Supplementary Figure 14). This result clearly shows that the small GO nanosheets were produced by breakage of the large GO nanosheets and that they were not produced during the oxidation of graphite powder. Thus, if small GO nanosheets with many carboxylic acid groups are needed for certain purposes, the graphite will need to be highly oxidised by step-by-step process before exfoliation. Furthermore, the real conductivities of LRGO and SRGO films measured by terahertz time-domain spectroscopy clearly show the decrease of the electrical property of graphene nanosheets prepared at the high energetic cavitation condition (Figs. $3 g$ and Supplementary Fig. 15).

The advantage of the less defective cavitation by probe sonication was also demonstrated by dispersing bundled single-walled carbon nanotubes (SWCNTs) in the aqueous surfactant solution. SWCNTs were dispersed by probe sonication varying the probe depth in a $1 \mathrm{wt} \%$ sodium dodecylbenzenesulphate solution to a SWCNT concentration of $1 \mathrm{~g} \mathrm{~L}^{-1}$. Figure $4 \mathrm{a}$ shows a plot of the sheet resistance $\left(R_{s}\right)$ versus transmittance $(T) . R_{s}$ and $T$ are related by ${ }^{23}$,

$$
T(\lambda)=\left(1+\frac{188.5}{R_{s}} \frac{\sigma_{O p}(\lambda)}{\sigma_{D C}}\right)^{-2}
$$

where $\sigma_{\mathrm{DC}}$ and $\sigma_{\mathrm{Op}}$ are the DC and optical conductivities, respectively. The ratio $\sigma_{\mathrm{DC}} / \sigma_{\mathrm{Op}}$ is usually treated as a figure of merit. Equation 1, combined with the values for $R_{s}$ and $T$, implied a value of $\sigma_{\mathrm{DC}} / \sigma_{\mathrm{Op}} \approx 12$ at a $2.5 \mathrm{~cm}$ probe depth. However, by adjusting the probe depth to the liquid surface, $R_{s}$ of the film decreased to less than $80 \%$ of the $R_{s}$ of the SWCNT film at a $0.5 \mathrm{~cm}$ probe depth. These values correspond to a $\sigma_{\mathrm{DC}} / \sigma_{\mathrm{Op}} \approx 15.7$. The $R_{s}$ changes of the SWCNT film after unusual probe sonication may be understood by considering the defect formation on the SWCNT surface during sonication. The electrical conductivity of the CNT networked films depends on the crystalline structure of the CNTs. The intensity ratio of the $\mathrm{G}$ and defect $\mathrm{D}$ bands $\left(I_{D} / I_{G}\right)$ in the Raman spectrum is related to the size of the $\mathrm{sp}^{2}$ carbon clusters in the CNTs and is nearly proportional to the defect density ${ }^{24}$. It is worth noting that the SWCNTs dispersed by probe sonication at a $0.5 \mathrm{~cm}$ probe depth showed a smaller $I_{D} / I_{G}$ value (indicating a less defective CNT structure) than SWCNTs dispersed by probe sonication at a $2.5 \mathrm{~cm}$ probe depth (Fig. 4b).

\section{Discussion}

Along with these experiments, we proved that layered materials can be extraordinarily exfoliated and dispersed in solution by controlling the acoustic cavitation, mechanical shearing, and microbubbling by probe sonicator. Our dispersion/exfoliation method allowed us to prepare suspensions of less defective, large 2D nanosheets or 1D nanotubes without the decomposition of used solvents molecules or nanosheets itself. These results provide new insights into the application of acoustic cavitation for the dispersion, exfoliation, and sonochemical reaction of nanomaterials. We propose that this cavitation technique can be applied to exfoliate and disperse graphite,

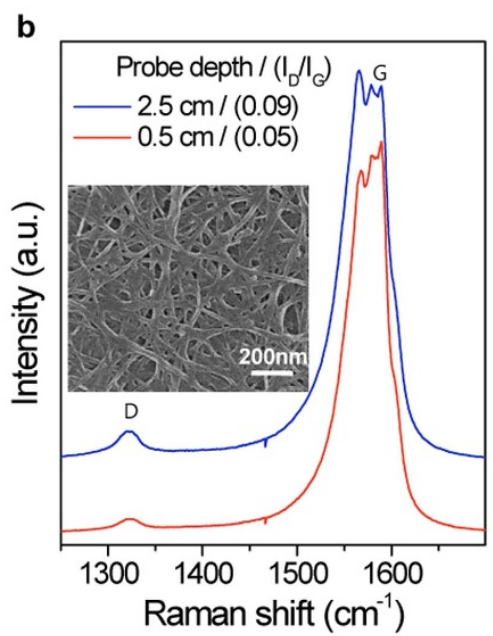

Figure 4 Dispersion of single-walled carbon nanotubes (SWCNTs) for transparent conducting films. (a) Transmittance (at $550 \mathrm{~nm}$ ) as a function of the sheet resistance for SWCNT films prepared by varying the probe depth during sonication. Inset plot: Transmittance versus sheet resistance data plotted to emphasize the goodness of fit to Eq. 1. (b) Raman spectra of the SWCNTs fabricated by probe sonication adjusting probe depth to 2.5 and $0.5 \mathrm{~cm}$ for $2 \mathrm{~h}$. The inset FESEM image shows the surface morphology of the SWCNT film. 
carbon nanotubes, other transition metal dichalcogenides, boron nitride, and other layered compounds in solvents.

\section{Methods}

Calibration of probe sonication. The probe type sonicator from Bandelin Electronics (SONOPULS HD2200, $20 \mathrm{kHz}, 200 \mathrm{~W}$ ) was used. The $12.7 \mathrm{~mm}$ diameter titanium probe tip was introduced in the sample. The amplitude of the ultrasound $(10-100 \%)$ and the immersion depth of the probe $(0.5 \mathrm{~cm}$ and $2.5 \mathrm{~cm}$ from the liquid surface) were varied in a standard $200 \mathrm{~mL}$ solution. The temperature and flow rate during probe sonication were measured by using a thermocouple and a flow rate sensor (Grinsinger, GMH 3330 with STS 005, Germany), which were separated from the probe tip by $1 \mathrm{~cm}$ (Supplementary Fig. 1). The power $(P)$ delivered into the solution by an acoustic probe was measured by the calorimetric method using the following equation:

$$
p=d T / d t \times C_{p} \times M
$$

where $T$ and $t$ are temperature (K) and time (s), respectively; $C_{p}$ is the specific heat capacity $(\mathrm{J} / \mathrm{g} / \mathrm{K})$; and $M$ is the mass of the liquid $(\mathrm{g})$.

Exfoliation and dispersion of $\mathrm{MoS}_{2}$ in solution. $\mathrm{MoS}_{2}$ powder (>99\% pure). Nmethyl-2-pyrrolidone, and dimethylsulphoxide were purchased from Sigma Aldrich and used as received. The decomposition of the solvents by probe sonication was monitored by measuring the UV-vis absorbance while varying the probe depth from the liquid surface to a $2.5 \mathrm{~cm}$ depth at different sonication times. The surface tension of the dispersing solvent was determined by the ring method with KRÜSS tensiometer. Exfoliation and dispersion of $\mathrm{MoS}_{2}$ in common solvents was performed at the same sonication conditions by adding $200 \mathrm{mg} \mathrm{MoS}$ powder to $200 \mathrm{~mL}$ of solvent in glass beakers under the cooling by a water bath because of the dramatic temperature increase during sonication. The dispersions were then centrifuged at 1500,5000 , and $10,000 \mathrm{rpm}$ for $20 \mathrm{~min}$.

Fabrication of graphene oxide (GO) nanosheets. Graphite oxide was synthesised using a modified Brodie method. Pure graphite (Alfa Aesar, $99.999 \%$ purity, -200 mesh) was mixed with fuming nitric acid and sodium chlorate at room temperature with stirring for $48 \mathrm{~h}$. After the acid treatment, the product was purified by washing, filtering, and cleaning. The synthesized graphite oxide powder was immersed in aqueous $\mathrm{KOH}$ with $\mathrm{pH}=10$ to a certain concentration. The exfoliation and dispersion was carried out using probe or bath sonicators. Subsequently, an aging process was performed to attach the $\mathrm{K}^{+}$ions onto the graphene basal plane using a rotary evaporator for $2 \mathrm{~h}$, and the excess $\mathrm{K}^{+}$ions were removed by several centrifugal washes. To obtain the dispersible GO powder, the resulting mixture was centrifuged and freeze-dried. Moreover, to prepare the conducting pastes, the resulting GO powder was then dispersed in dimethylformamide (DMF) by vigorous shaking and sonication. To fabricate conducting pastes, the GO nanosheets dispersed in DMF were reacted with toluene diisocyanate and 2-amino-4-hydroxy-6-methylpyrimidine to attach the 2 -ureido- $4[1 \mathrm{H}]$ pyrimidinone moieties forming quadruple hydrogen bonding $(\mathrm{QHB})$ networks as previously reported ${ }^{22}$. The GO paste was reduced by adding hydrazine monohydrate $\left(\mathrm{N}_{2} \mathrm{H}_{4}\right)$ to reach a final concentration of $4 \mathrm{mM}$, followed by heating at $100^{\circ} \mathrm{C}$ for $10 \mathrm{~h}$.

Dispersion of single-walled carbon nanotubes (SWCNTs) and fabrication of transparent conducting films. SWCNTs produced by the arc-discharge method and purified by thermal treatment (purchased from Nanosolutions, Extube) were used in this study. The SWCNTs were dispersed by probe sonication in a $1 \mathrm{wt} \%$ sodium dodecylbenzenesulphate solution to a concentration of $1 \mathrm{~g} / \mathrm{L}$ for $2 \mathrm{hr}$, followed by two rounds of centrifugation at $10000 \mathrm{rpm}$ for $60 \mathrm{~min}$. The supernatant solution was deposited onto polyethylene terephthalate films using an automatic spray coater (NCS-400) with a 1.2-mm diameter nozzle. The surfactant was removed by dipping in deionised water twice for $10 \mathrm{~min}$ each, followed by air drying at $70^{\circ} \mathrm{C}$ for $10 \mathrm{~min}$.

Characterisation. UV-vis-IR absorption spectroscopy was performed with a Cary 5000 using $1 \mathrm{~cm}$ quartz cuvettes. The morphologies of the samples were imaged by field-emission scanning electron microscopy (FESEM, HITACHI S4800) and atomic force microscopy (AFM, Digital Instruments Nanoscope IIIA) using silicon tips with a typical resonance frequency of $320 \mathrm{kHz}$. Transmission electron microscopy (TEM) was carried out with a FEI TECHNAI G2 F20, which was operated at an acceleration voltage of $120 \mathrm{kV}$. The structural characteristics of chemically-modified graphene nanosheets, and SWCNTs were investigated by confocal Raman spectrometry (NTEGRA SPECTRA, NT-MDT) with excitation wavelengths of 532 and $633 \mathrm{~nm}$. The functional groups of the GO nanosheets were assigned by X-ray photoelectron spectroscopy (XPS) using a Multilab2000 (Thermo VG Scientific Inc.) spectrometer with monochromatised Al K X-ray radiation as the X-Ray excitation source. The stability of the $\mathrm{GO}$ and $\mathrm{MoS}_{2}$ dispersions was tested by observing the sedimentation behavior under centrifugation at $1500 \mathrm{rpm}$ using a LUMiSizer ${ }^{\circledR}$ LS611 (L.U.M. $\mathrm{GmbH}$, Berlin, Germany), which is a microprocessor-controlled analytical centrifuge allowing the determination of space- and time-resolved transmission profiles during the centrifugation. To characterise the colloidal behaviour in a liquid droplet, GO suspensions in water with a concentration of $500 \mathrm{mg} / \mathrm{L}$ were ink-jet printed on the $\mathrm{SiO}_{2}$ substrates and treated with UV-ozone for $10 \mathrm{~min}$. A home-built ink-jet printer was equipped with a single-nozzle drop-on-demand piezoelectric print head (Microfab Jet Drive III), a two-axis motorised positioning system, and a CCD camera aligned with a LED for the visualisation of the droplet ejection. Single droplets with volumes of $40-50 \mathrm{pL}$ were ejected on demand from the nozzle with a diameter of $100 \mu \mathrm{m}$. The vertical separation between the nozzle and the substrate was typically $0.5 \mathrm{~mm}$.

1. Suslick, K. Sonochemistry. Science 23, 1439-1445 (1990)

2. Shchukin, D. G., Skorb, E., Belova, V. \& Möhwald, H. Ultrasonic Cavitation at Solid Surfaces. Adv. Mater. 23, 1922-1934 (2011).

3. Xu, H., Zeiger, B. W. \& Suslick, K. S. Sonochemical synthesis of nanomaterials. Chem. Soc. Rev. 42, 2555-2567 (2013)

4. O'Brien Jr., W. D. Ultrasound-biophysics mechanisms. Prog. Biophys. Mole. Biol. 93, 212-255 (2007)

5. Suslick, K. S. Ultrasound: its chemical, physical, and biological effects. (New York: VCH Publishers, 1988).

6. Mason, T. J. \& Lorimer, J. P. Applied Sonochemistry: The uses of power ultrasound in chemistry and processing (Wiley-VCH, Weinheim, 2002).

7. Chen, D., Sharma, K. \& Mudhoo, A. Handbook on applications of ultrasound: Sonochemistry for sustainability (CRC Press, 2011).

8. Hernandez, Y. et al. High-yield production of graphene by liquid-phase exfoliation of graphite. Nature Nanotech. 3, 563-568 (2008).

9. Park, S. \& Ruoff, R. S. Chemical methods for the production of graphenes. Nature Nanotech. 4, 217-224 (2009).

10. Coleman, J. N. et al. Two-dimensional nanosheets produced by liquid exfoliation of layered materials. Science 331, 568-571 (2011).

11. Chhowalla, M. et al. H. The chemistry of two-dimensional layered transition metal dichalcogenide nanosheets. Nature Chem. 5, 263-275 (2013).

12. Nocolosi, V. et al. Liquid exfoliation of layered materials. Science DOI:10.1126/ science.1226419.

13. Flynn, H. G. Physics of acoustic cavitation in liquids, in: Physical Acoustics Vol. 1, Part B 57-172 (Ed. Mason, W. P., Academic Press, New York, USA, 1964).

14. Jeong, S. Y. et al. High-performance transparent conductive films using rheologically derived reduced graphene oxide. ACS Nano 5, 870-878 (2011).

15. Margulis, M. A. \& Margulis, I. M. Calorimetric method for measurement of acoustic power absorbed in a volume of a liquid. Ultrasonics Sonochem. 10, 343-345 (2003).

16. Louisnard, O. et al. High bubble concentrations produced by ultraounds in binary mixtures. Ultrasonics Sonochem. 8, 183-189 (2001).

17. Thyrion, F. C. \& Debecker, G. Thermolysis of dimethyl sulfoxide. Int. J. Chem. Kinetics 5, 583-592 (1973).

18. White, A. M., Grove, B. \& Sankey, B. M. Thermal stabilization of N-methyl-2pyrrolidone, U.S. Patent 4168226 (1979).

19. Li, J. L. et al. Oxygen-driven unzipping of graphitic materials. Phys. Rev. Lett. 96, 176101 (2006).

20. Ajayan, P. M. \& Yakobson, B. I. Materials Science-Oxygen breaks into carbon World. Nature 441, 818-819 (2006).

21. Wang, H., Robinson, J. T., Li, X. \& Dai, H. Solvothermal reduction of chemically exfoliated graphene sheets. J. Am. Chem. Soc. 131, 9910-9911 (2009).

22. Han, J. T. et al. Dispersant-free conducting pastes for flexible and printed nanocarbon electrodes. Nature Commun. DOI: 10.1038/ncomms3491.

23. Hu, L., Hecht, D. S. \& Grüner, G. Percolation in Transparent and Conducting Carbon Nanotube Networks. Nano Lett. 4, 2513-2517 (2004).

24. Yang, L. \& Han, J. Electronic Structure of Deformed Carbon Nanotubes. Phys. Rev. Lett. 85, 154-157 (2000).

\section{Acknowledgments}

This work was supported by the Center for Advanced Soft-Electronics funded by the Ministry of Science, ICT and Future Planning as Global Frontier Project (2013M3A6A5073177), and by Primary Research Program of Korea Electrotechnology Research Institute, South Korea.

\section{Author contributions}

J.T.H. conceived and supervised the experiment and wrote the manuscript. J.I.J. calibrated the probe sonication and performed the liquid exfoliation. S.C., H.K., S.J. and H.Y.K. performed the characterization of the nanosheets. J.Y.H. carried out the transmission electron spectroscopy imaging, H.K.Y. measured time-domain signals of THz transmission, and J.S.W. fabricated the SWCNT films. H.J.J., S.Y.J. and K.-J.B. analyzed the data. K.C. and G.-W.L. supervised the experiment and discussed the data. All authors discussed the results and commented on the manuscript.

\section{Additional information}

Supplementary information accompanies this paper at http://www.nature.com/ scientificreports

Competing financial interests: The authors declare no competing financial interests. 
How to cite this article: Han, J.T. et al. Extremely Efficient Liquid Exfoliation and Dispersion of Layered Materials by Unusual Acoustic Cavitation. Sci. Rep. 4, 5133; DOI:10.1038/srep05133 (2014)

(c) (i) $(-)$ This work is licensed under a Creative Commons Attribution-NonCommercial(c) ${ }_{\mathrm{Br}} \mathrm{NC}$ ND NoDerivs 3.0 Unported License. The images in this article are included in the article's Creative Commons license, unless indicated otherwise in the image credit; if the image is not included under the Creative Commons license, users will need to obtain permission from the license holder in order to reproduce the image. To view a copy of this license, visit http://creativecommons.org/licenses/by-nc-nd/3.0/ 